\title{
Resultados de cirugía combinada, mSICS y ciclodiálisis, en pacientes con glaucoma y catarata. Experiencia, eficacia y seguridad
}

\section{Results of combined surgery, mSICS and cyclodialysis, in patients with glaucoma and cataract. Real-life experience, efficacy and safety}

\author{
Mario R. Papa-Vettorazzi ${ }^{1 *}$, José B. Cruz-Rodríguez², Claudia M. López-Villeda y Gladys L. Silva-Linares ${ }^{1}$ \\ ${ }^{1}$ Visualiza Clinic, Guatemala, Guatemala; ${ }^{2}$ Texas Tech University Health Sciences Centre, El Paso, Texas, Estados Unidos de América
}

\section{Resumen}

Objetivo: Evaluar la seguridad y la eficacia de la cirugía combinada (cirugía manual de incisión pequeña [mSICS] y ciclodiálisis) en ojos con catarata y glaucoma avanzado o hipertensión ocular no controlada. Método: Estudio retrospectivo de 343 ojos consecutivos en los que se realizaron mSICS y ciclodiálisis. Todas las cirugías fueron llevadas a cabo durante 2017 y 2018 por cirujanos experimentados. Para evaluar la eficacia y la seguridad del procedimiento se usó un grupo control de 53 ojos consecutivos a los que solo se realizó ciclodiálisis durante el mismo periodo. En ambos grupos se evaluaron y compararon el cambio en la presión intraocular (PIO), los medicamentos para el control del glaucoma, la agudeza visual mejor corregida (AVMC), las complicaciones y las reintervenciones. Resultados: La disminución de la PIO fue mayor en el grupo de cirugía combinada $(p=0.01)$, la necesidad de medicamentos para el control del glaucoma fue menor en el grupo de cirugía combinada $(p=0.07) y$, del mismo modo, la AVMC fue mejor en este grupo $(p=0.05)$. La frecuencia de complicaciones fue menor en el grupo de ciclodiálisis $(p=0.03)$. La frecuencia de reintervenciones fue menor en el grupo de cirugía combinada $(p<0.01)$. En ambos grupos, la mayoría de las reintervenciones fueron procedimientos para controlar la $P I O$. El éxito y el éxito calificado fueron superiores en el grupo de cirugía combinada $(p<0.01)$. Conclusiones: La cirugía de mSICS y ciclodiálisis es efectiva y relativamente segura en ojos con catarata y glaucoma avanzado o con hipertensión ocular no controlada. Es un procedimiento de bajo costo que representa una buena alternativa en los países en vías de desarrollo.

Palabras clave: Catarata. Ciclodiálisis. Cirugía combinada. Glaucoma. mSICS.

\begin{abstract}
Objective: To assess the efficacy and safety of combined surgery (manual small incision cataract surgery [mSICS] and cyclodialysis) in eyes with cataracts and advanced glaucoma or uncontrolled ocular hypertension. Method: Retrospective study of 343 consecutive eyes that underwent mSICS and cyclodialysis. All surgeries were performed by experienced surgeons during 2017 and 2018. To evaluate the efficacy and safety of the procedure, we used a control group of 53 consecutive eyes that only underwent cyclodialysis during the same period. In both groups, the change in intraocular pressure (IOP),
\end{abstract}

Correspondencia:

*Mario R. Papa-Vettorazzi

5ta Avda. 11-44, Zona 9

Fecha de recepción: 19-01-2021

Fecha de aceptación: 12-05-2021

C.P. 01009, Guatemala, Guatemala

E-mail: mrpv_papa@hotmail.com

DOI: 10.24875/RMO.M21000183

0187-4519/@ 2021 Sociedad Mexicana de Oftalmología. Publicado por Permanyer. Este es un artículo open access bajo la licencia CC BY-NC-ND (http://creativecommons.org/licenses/by-nc-nd/4.0/).
Disponible en internet: 01-09-2021 Rev Mex Oftalmol. 2021;95(5):179-187

www.rmo.com.mx 
glaucoma control medications, best-corrected visual acuity (BCVA), complications, and reinterventions were evaluated and compared. Results: The decrease in IOP was greater in the combined surgery group $(p=0.01)$. The need for medications to control glaucoma was lower in the combined surgery group $(p=0.07)$ and, in the same way, BCVA was better in this group $(p=0.05)$. The frequency of complications was lower in the cyclodialysis group $(p=0.03)$. The frequency of reoperations was lower in the combined surgery group $(p<0.01)$. In both groups, most reoperations were procedures to control IOP. Success and graded success were higher in the combined surgery group $(p<0.01)$. Conclusions: Combined mSICS surgery is effective and relatively safe in eyes with cataracts and advanced glaucoma or with uncontrolled ocular hypertension. It is a low-cost procedure that represents a good alternative in developing countries.

Keywords: Cataract. Cyclodialysis. Combined surgery. Glaucoma. mSICS.

\section{Introducción}

Las cataratas y el glaucoma son las dos causas más comunes de ceguera prevenible en todo el mundo ${ }^{1}$. A pesar de los avances tecnológicos, estas condiciones aún representan un importante problema de salud pública, en especial en los países de bajos ingresos, donde el acceso a la atención especializada y el costo del tratamiento son una barrera importante.

El tratamiento de las cataratas es quirúrgico, mientras que el del glaucoma puede ser médico, con láser o quirúrgico, según la gravedad. Cuando existen ambas condiciones, el abordaje quirúrgico es ampliamente debatido, con varios autores que abogan por realizar los procedimientos en un orden específico y muchos otros que favorecen la realización de ambos de manera simultánea ${ }^{2-5}$.

El procedimiento combinado de referencia es la facoemulsificación más trabeculectomía. Sin embargo, en poblaciones de bajos ingresos este tipo de cirugía tiene ciertas desventajas, derivadas principalmente del costo y el seguimiento.

Hay algunos informes que concluyen que la combinación de extracción de catarata más ciclodiálisis tiene éxito en reducir la presión intraocular (PIO) y los medicamentos para el glaucoma posoperatoriamente ${ }^{6,7}$. Esta es la razón por la que la cirugía manual de catarata con incisión pequeña (mSICS, manual small-incision cataract surgery) más ciclodiálisis puede ser una buena alternativa.

El objetivo principal de este estudio fue evaluar la eficacia y la seguridad de la ciclodiálisis combinada con mSICS en comparación con la ciclodiálisis sola. Los objetivos secundarios fueron los cambios a corto y largo plazo en la PIO, los cambios en los requerimientos de medicamentos para el glaucoma, los cambios en la agudeza visual mejor corregida (AVMC) a los 3 meses, las complicaciones y las reintervenciones.

\section{Método}

\section{Diseño del estudio}

Realizamos un estudio de cohorte retrospectivo de un solo centro, que incluyó 343 ojos consecutivos sometidos a cirugía combinada (mSICS más ciclodiálisis). Los procedimientos fueron realizados por cirujanos experimentados utilizando una técnica estándar durante 2017 y 2018. Todos los ojos tenían una catarata que causaba alteración visual significativa (agudeza visual peor que 0.3 logMar) y glaucoma avanzado no controlado o hipertensión ocular no controlada (PIO superior a la meta considerada para cada caso a pesar de la terapia médica o láser, según la evaluación clínica).

Para evaluar la eficacia y la seguridad del procedimiento utilizamos un grupo control de 53 ojos consecutivos que se sometieron a ciclodiálisis sola (ojos fáquicos y pseudofáquicos), realizada por el mismo grupo de cirujanos durante el mismo periodo.

En ambos grupos, la consideración del daño al nervio óptico y la clasificación del glaucoma se hicieron según la evaluación clínica y la PIO preoperatoria (tonómetro de Goldman).

Uno de los investigadores hizo una revisión manual de la historia y otro la validó. Las variables clínicas extraídas en ambos grupos fueron edad, sexo, ojo, tipo de glaucoma, excavación (evaluación clínica), fecha del procedimiento y último seguimiento. Las variables de resultados fueron la PIO (tonómetro de Goldman), el número de medicamentos para el glaucoma en el día 1 y tras 1 semana, 1, 3, 6 y 12 meses, la AVMC posoperatoria a los 3 meses y el fracaso (si ocurría).

Un comité de ética institucional externo confirmó la exención de este trabajo, dado su carácter retrospectivo, y aprobó el estudio. La investigación cumplió los principios de la Declaración de Helsinki.

Debido a que algunas agudezas visuales se expresaron en términos no numéricos, se les asignó un rango/valor en escala logMar para su análisis (Anexo 1). 
Se realizó ultrasonido A/B antes de la cirugía a todos los pacientes con catarata madura que no permitía la evaluación fundoscópica.

\section{Procedimiento quirúrgico}

Después de la sedación administrada por un anestesiólogo y la aplicación de anestesia peribulbar (mezcla 1:1 de lidocaína al 1\%, bupivacaína al $0.75 \%$ y 150 unidades de hialuronidasa), los pacientes fueron preparados para la cirugía de la forma habitual. Se colocó un espéculo palpebral, se realizó una peritomía basada en el fórnix de $6 \mathrm{~mm}$ seguida de diatermia ligera para campo húmedo en los vasos epiesclerales. Se realizó una incisión escleral de 5.5-6 $\mathrm{mm}$ con una hoja de diamante 0 un bisturí de $15^{\circ}$ comenzando $1-2 \mathrm{~mm}$ detrás del limbo (el cirujano decidió la ubicación de la peritomía y de la incisión escleral de acuerdo con la queratometría preoperatoria). Esta incisión se realizó con una cuchilla Crescent hasta que se alcanzó el limbo, avanzando 1-1.5 mm hacia la córnea clara. Se realizó paracentesis con una hoja de queratomo de $3.2 \mathrm{~mm}$. En algunos casos, los cirujanos utilizaron azul tripán para teñir la cápsula anterior, y luego la cámara anterior se llenó con viscoelástico. Posteriormente, se abrió todo el labio interno de la incisión en túnel en forma de válvula con la hoja de queratomo de $3.2 \mathrm{~mm}$. A continuación, se realizó una rexis capsular mediante un quistitomo. Se llevó a cabo hidrodisección y luxación de la catarata a la cámara anterior para extracción de la catarata mediante una cánula de irrigación Vectis. Todo el material cortical se extrajo utilizando una cánula Simcoe de 21. Luego se introdujo una lente de polimetilmetacrilato de una sola pieza en la bolsa capsular. A continuación, se utilizó un miótico y se realizó una hendidura de ciclodiálisis de dos meridianos con la espátula de ciclodiálisis (ubicación determinada por el cirujano).

Para realizar la ciclodiálisis se introdujo la espátula a través de la paracentesis en el cuadrante opuesto a donde se realizaría. Se realizó un movimiento superficial inicial para efectuar una goniotomía. Seguidamente, mientras se mantenía una ligera elevación en la esclerótica, se siguió la córnea periférica con la espátula para alcanzar el espolón escleral. Luego se presionó suavemente la punta de la espátula y se dejó pasar más allá del espolón escleral hacia el espacio supraciliar. Una vez realizada la ciclodiálisis, se observó la cámara anterior durante 102 minutos para detectar cualquier hemorragia. Por último, se retiró el viscoelástico, se hidrató la paracentesis y se revisó la herida principal en busca de fugas. La conjuntiva se cerró utilizando diatermia o una sutura de nailon 10-0.

En el grupo de ciclodiálisis sola, la técnica quirúrgica fue similar. Después de la sedación, la administración de anestesia peribulbar y la preparación estándar del paciente, se realizó la paracentesis con una cuchilla de $3.2 \mathrm{~mm}$ (ubicación decidida por el cirujano). Se utilizó un miótico, la cámara anterior se llenó de viscoelástico y se realizó ciclodiálisis de dos meridianos utilizando la misma técnica descrita para el grupo de cirugía combinada (ubicación determinada por el cirujano). Se observó la cámara anterior durante 102 minutos, luego se retiró el viscoelástico y se hidrató la paracentesis.

En todos los ojos se colocó parche y protector una vez finalizado el procedimiento.

\section{Cuidado posoperatorio}

Los pacientes utilizaron gotas combinadas de antibióticos y esteroides cada 102 horas mientras estaban despiertos durante 7 días, que luego se disminuyeron lentamente durante 1 mes, a menos que la inflamación no se resolviera. En algunos casos se utilizó pilocarpina al 4\% tres veces al día para evitar el cierre de la ciclodiálisis. El seguimiento fue el primer día, luego semanal durante el primer mes, y después a los 3, 6 y 12 meses.

\section{Definiciones de éxito, éxito calificado y fracaso}

El control de la PIO se utiliza a menudo como una medida sustituta de la estabilidad de la enfermedad en los estudios de cirugía de glaucoma. Sin embargo, no existen unas definiciones claras y universalmente aceptadas de éxito y de éxito calificado ${ }^{8}$.

La PIO meta más estricta informada en la mayoría de los estudios es $\leq 18 \mathrm{mmHg}^{2,6}$. Sin embargo, consideramos que en los pacientes con glaucoma avanzado el objetivo debe ser obtener unos valores de PIO más bajos. El Advanced Glaucoma Intervention Study (AGIS) informó de que la PIO con la cual la pérdida del campo visual se estabiliza es, en promedio, de $12.3 \mathrm{mmHg}$, y recomienda también reducciones meta desde la basal del $40 \%$ en los casos graves o del $30 \%$ en los moderados 9 .

Por eso, realizamos dos análisis separados. En el primero aplicamos la definición de PIO $<18 \mathrm{mmHg}$ : - Éxito: PIO < $18 \mathrm{mmHg}$ sin medicación para el glaucoma. 
- Éxito calificado: PIO < 18 mmHg con medicación para el glaucoma.

- Fracaso: $\mathrm{PIO} \geq 18 \mathrm{mmHg}$ o necesidad de procedimientos subsecuentes para controlar la PIO.

En el otro aplicamos la definición sugerida por el AGIS (utilizada para el análisis de sensibilidad) ${ }^{9}$ :

- Éxito: PIO $\leq 12.3 \mathrm{mmHg}$, o una reducción del $40 \%$ en los casos graves, o una reducción del $30 \%$ en los casos moderados, sin medicación para el glaucoma.

- Éxito calificado: $\mathrm{PIO} \leq 12.3 \mathrm{mmHg}$, o una reducción del $40 \%$ en los casos graves, o una reducción del $30 \%$ en los casos moderados, con medicación para el glaucoma.

- Fracaso: PIO > $12.3 \mathrm{mmHg}$, o una reducción inferior al $40 \%$ en los casos graves, o una reducción inferior al $30 \%$ en los casos moderados, o necesidad de procedimientos subsecuentes para controlar la PIO.

\section{Análisis estadístico}

Se realizó un análisis descriptivo para todas las variables continuas. Las variables con distribución normal se presentan con media y desviación estándar, y las que no tenían distribución normal se presentan con mediana y rango intercuartílico. Para todas las variables categóricas presentamos frecuencias y proporciones. Según la distribución de los datos, se utilizó la prueba $t$ pareada o la prueba no paramétrica de Kruskal-Wallis para comparar las variables continuas. Los resultados categóricos se analizaron mediante la prueba de ji al cuadrado o la prueba exacta de Fisher cuando la muestra era más pequeña. Los valores de $p \leq 0.05$ se consideraron estadísticamente significativos. El análisis estadístico se basó en un nivel de significancia bilateral. Los datos faltantes se manejaron con el método de datos disponibles. Se realizó análisis de supervivencia de Kaplan-Meier para ambos grupos y se aplicó el análisis de regresión de Cox con método de Breslow para los empates a las variables clínicamente relevantes seleccionadas a priori. Los análisis se llevaron a cabo con STATA versión 14.2 (StataCorp LCC, Tx, USA).

\section{Resultados}

\section{Eficacia}

El grupo de cirugía combinada incluyó 343 ojos consecutivos, de los que el $92 \%$ completó al menos 1 mes de seguimiento, el $65 \%$ logró al menos un seguimiento de 3 meses, el $51 \%$ un seguimiento de 6 meses y el
Tabla 1. Características demográficas $(n=396)$

\begin{tabular}{|c|c|c|c|}
\hline Variable & $\begin{array}{l}\text { mSICS + } \\
\text { ciclodiálisis }\end{array}$ & Ciclodiálisis & $\mathbf{p}$ \\
\hline $\mathrm{n}$ & 343 & 53 & \\
\hline Edad, media (DE) & 73.43 (9.79) & 71.69 (14.38) & 0.26 \\
\hline Sexo masculino & $152(44.3 \%)$ & $29(54.7 \%)$ & 0.16 \\
\hline Ojo derecho & $170(49.6 \%)$ & $28(52.8 \%)$ & 0.66 \\
\hline $\begin{array}{l}\text { Excavación, media } \\
\text { (DE) }\end{array}$ & $0.795(0.178)$ & $0.858(0.148)$ & 0.01 \\
\hline $\begin{array}{l}\text { Tipo de glaucoma } \\
\text { GPAA } \\
\text { GCAC } \\
\text { PXG } \\
\text { Otro* }\end{array}$ & $\begin{array}{l}153(45.4 \%) \\
94(27.9 \%) \\
49(14.5 \%) \\
41(12.2 \%)\end{array}$ & $\begin{array}{c}15(29.4 \%) \\
5(9.8 \%) \\
7(13.7 \%) \\
24(47.1 \%)\end{array}$ & $<0.01$ \\
\hline $\begin{array}{l}\text { Localización de la } \\
\text { ciclodiálisis } \\
\text { SN } \\
\text { IN } \\
\text { ST } \\
\text { IT }\end{array}$ & $\begin{array}{c}205(60.8 \%) \\
65(19.3 \%) \\
28(8.3 \%) \\
39(11.6 \%)\end{array}$ & $\begin{array}{c}27(51.9 \%) \\
7(13.5 \%) \\
14(26.9 \%) \\
4(7.7 \%)\end{array}$ & $<0.01$ \\
\hline $\begin{array}{l}\text { AVMC logMar } \\
\text { preoperatoria, media } \\
\text { (DE) }\end{array}$ & $0.965(0.619)$ & $0.848(0.671)$ & 0.20 \\
\hline $\begin{array}{l}\text { Medicamentos } \\
\text { preoperatorios, media } \\
\text { (DE) }\end{array}$ & $1.57(1.40)$ & $2.37(1.27)$ & $<0.01$ \\
\hline $\begin{array}{l}\text { PIO preoperatoria, } \\
\text { media (DE) }\end{array}$ & $20.14(7.79)$ & $23.15(7.05)$ & 0.01 \\
\hline $\begin{array}{l}\text { AVMC logMar } \\
\text { posoperatoria, media } \\
\text { (DE) }\end{array}$ & $0.445(.461)$ & $0.622(.706)$ & 0.05 \\
\hline
\end{tabular}

AVMC: agudeza visual mejor corregida; DE: desviación estándar; GCAC: glaucoma crónico de ángulo cerrado; GPAA: glaucoma primario de ángulo abierto; IN: inferonasal; IT: inferotemporal; mSICS: cirugía manual de catarata con incisión pequeña; PIO: presión intraocular; PXG: glaucoma por pseudoexfoliación; SN: superonasal; ST: superotemporal.

*Hipertensión ocular, glaucoma secundario a esteroides, glaucoma por mecanismo combinado, glaucoma pigmentario.

$35 \%$ un seguimiento de 12 meses. El grupo de ciclodiálisis sola incluyó 53 ojos consecutivos, de los que el $91 \%$ completó al menos un mes de seguimiento, el $68 \%$ logró al menos un seguimiento de 3 meses, el $55 \%$ un seguimiento de 6 meses y el $39 \%$ un seguimiento de 12 meses. El seguimiento medio fue de 266 días para el grupo de cirugía combinada y de 231 días para el grupo de ciclodiálisis sola. Las características demográficas se presentan en la tabla 1.

La reducción de la PIO fue constante y significativa en ambos grupos; sin embargo, en el seguimiento final, el cambio fue mayor en el grupo de cirugía combinada $(p=0.01)$. En comparación absoluta, los ojos con cirugía combinada tuvieron una reducción de la PIO de 


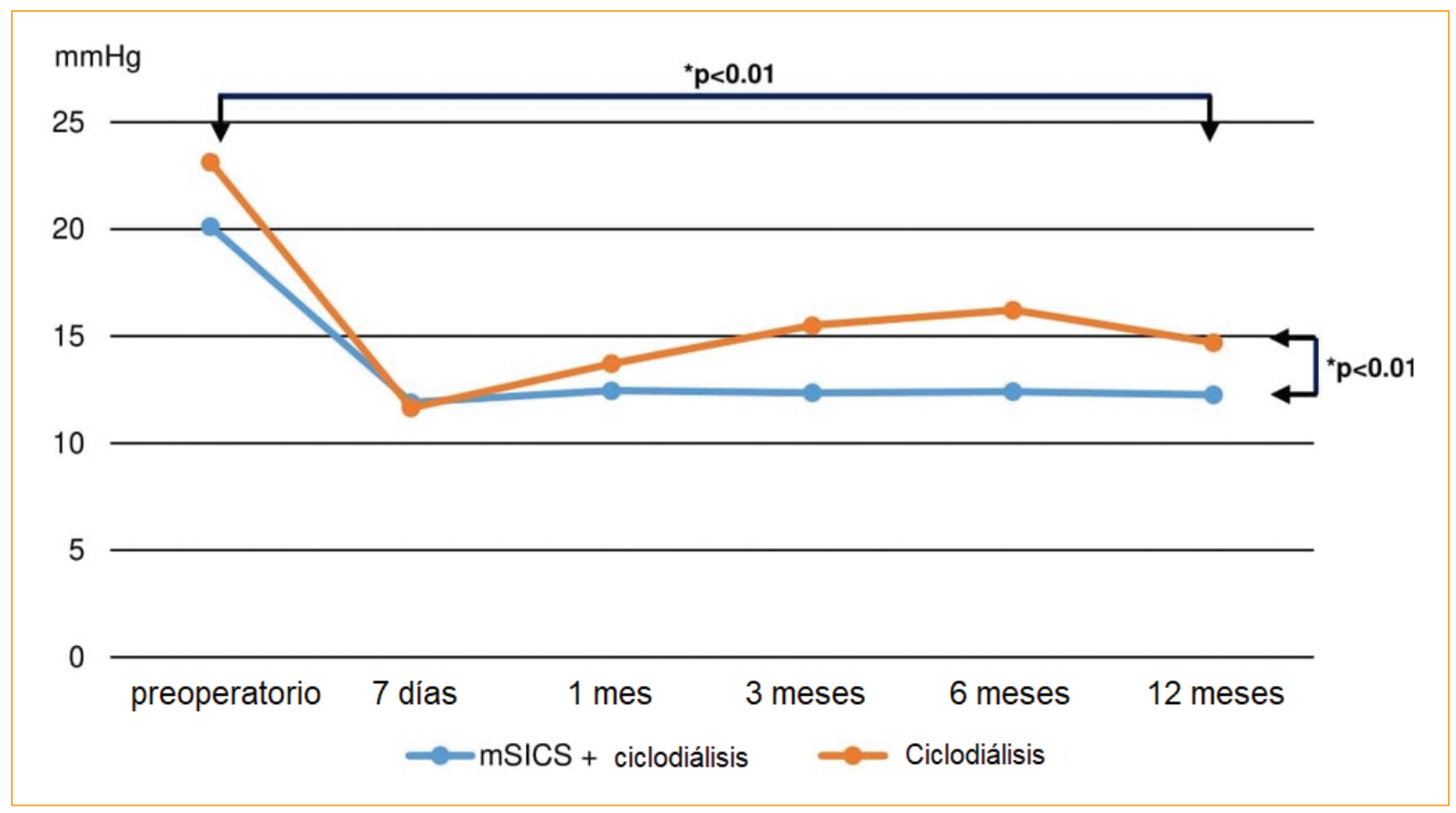

Figura 1. Gráfico de líneas que representa el cambio en la presión intraocular (PIO) a lo largo del tiempo y la comparación entre ambos grupos del estudio: cirugía combinada $(n=343)$ o ciclodiálisis sola $(n=53)$. Los valores de p corresponden a una prueba t pareada para cada grupo individual que compara la PIO preoperatoria con la PIO en la evaluación a los 12 meses, y una prueba t para comparar ambos grupos. mSICS: cirugía manual de catarata con incisión pequeña.

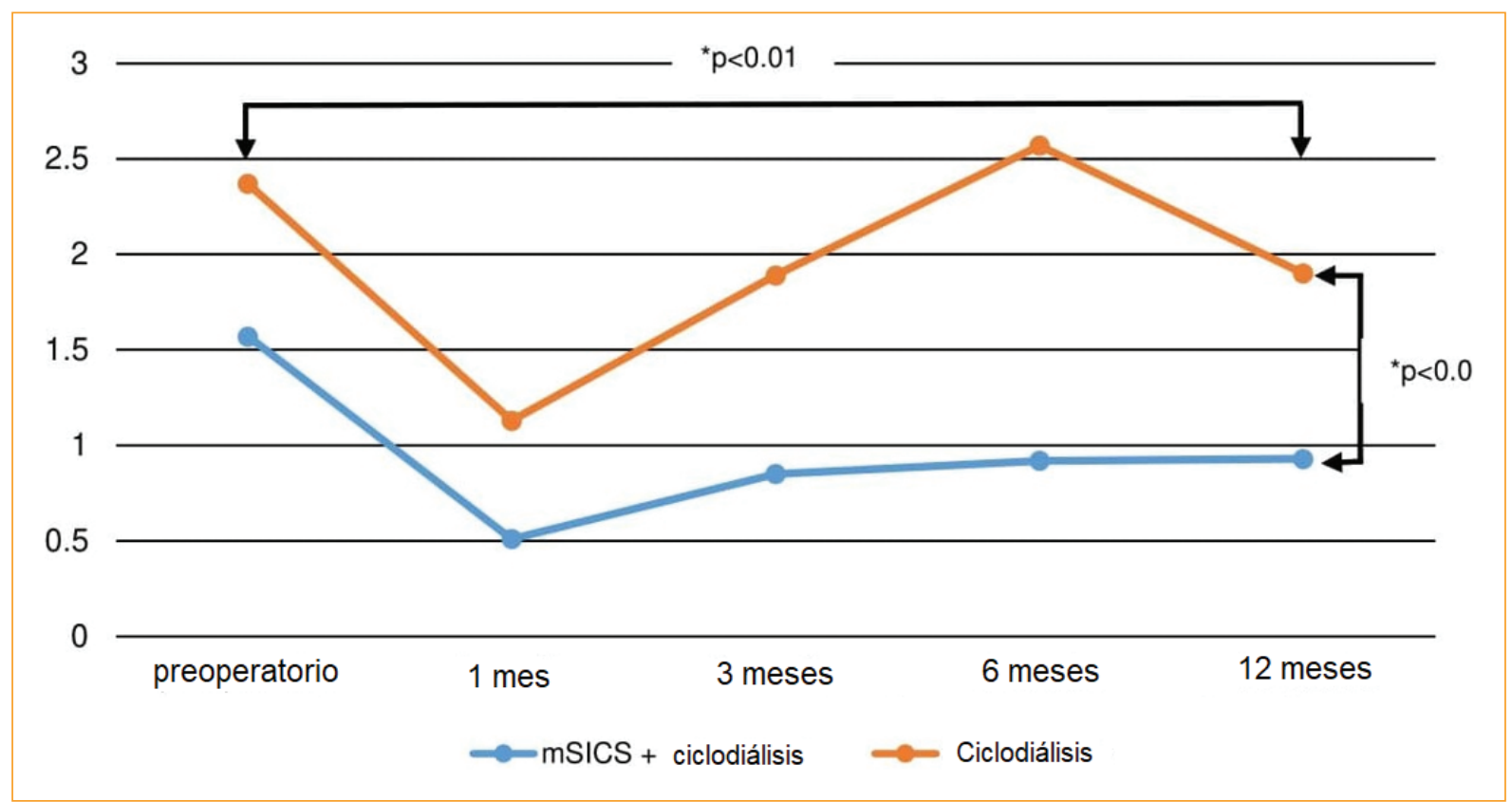

Figura 2. Gráfico de líneas que representa la reducción en la necesidad de medicamentos tras operación de glaucoma y la comparación entre ambos grupos del estudio: cirugía combinada $(n=343) 0$ ciclodiálisis sola ( $n=$ 53). Los valores $p$ corresponden a una prueba t pareada para cada grupo individual que compara los requisitos de medicación preoperatoria con los requisitos de medicación en la evaluación de 12 meses, y una prueba t para comparar ambos grupos. mSICS: cirugía manual de catarata con incisión pequeña. 
$3.72 \mathrm{mmHg}$ (intervalo de confianza del 95\% [IC95\%]: -1.36 a -6.07; $p<0.01$ ). La diferencia media de la PIO preoperatoria y posoperatoria a los 12 meses de seguimiento fue de $7.06 \mathrm{mmHg}( \pm 8.5)$ en el grupo de cirugía combinada y de $5.33 \mathrm{mmHg}( \pm 11.02)$ en el grupo de ciclodiálisis sola (Fig. 1).

Aunque el grupo de cirugía combinada necesitó menos medicamentos antes de la cirugía, en el último seguimiento el número de medicamentos requeridos fue de 0.78 menos en comparación con el grupo de ciclodiálisis sola $(p<0.01)$. La disminución media de los requisitos de medicación para el glaucoma en el seguimiento de 12 meses fue de $0.576( \pm 1.33)$ en el grupo de cirugía combinada y de $0.476( \pm 1.96)$ en el grupo de ciclodiálisis sola (Fig. 2).

Aplicando la definición de $<18 \mathrm{mmHg}$, el grupo de cirugía combinada no alcanzó la mediana de tiempo hasta el fracaso al final del seguimiento; alcanzó el percentil 75 a los 620 días (1.7 años). La mediana de tiempo hasta el fracaso para el grupo de ciclodiálisis sola fue de 243 días (8 meses). Las tasas de éxito calificadas al final del primer año fueron del $83.4 \%$ en el grupo de cirugía combinada y del $46.8 \%$ en el grupo de ciclodiálisis sola (rango logarítmico de Wilcoxon, $p<0.01$ ) (Fig. 3).

Aplicando la definición del AGIS, el grupo de ciclodiálisis sola tuvo una tasa de fracaso más alta en cada momento de tiempo, con una mediana de tiempo hasta el fracaso de 166 días (5.5 meses), en comparación con una mediana de tiempo hasta el fracaso de 747 días (2.05 años) en el grupo de cirugía combinada. Las tasas de éxito calificado al final del primer año fueron del $66.1 \%$ en el grupo de cirugía combinada y del $36.7 \%$ en el grupo de ciclodiálisis sola (rango logarítmico de Wilcoxon, $\mathrm{p}<0.01$ ) (Fig. 4).

Las tasas de éxito y de éxito calificado aplicando ambas definiciones fueron significativamente mejores en el grupo de cirugía combinada, incluso cuando se aplicó la definición más estricta (Tabla 2).

Se aplicó la regresión de Cox con el objetivo de predecir los eventos. En un análisis crudo, el riesgo de desarrollar un evento fue un $49 \%$ menor (hazard ratio [HR]: $0.51 ; p=0.002$; IC95\%: 0.34 a 0.84) en los pacientes que se sometieron a cirugía combinada en comparación con aquellos en quienes solo se realizó ciclodiálisis.

Después de ajustar para las variables seleccionadas a priori dado su impacto clínico en el resultado (diagnóstico, localización de la ciclodiálisis y PIO basal), el riesgo de desarrollar un evento fue un $67 \%$ menor (HR: 0.33; $p<0.01$; IC95\%: 0.20 a 0.57) en los pacientes sometidos a cirugía combinada en comparación con los tratados solo con ciclodiálisis.

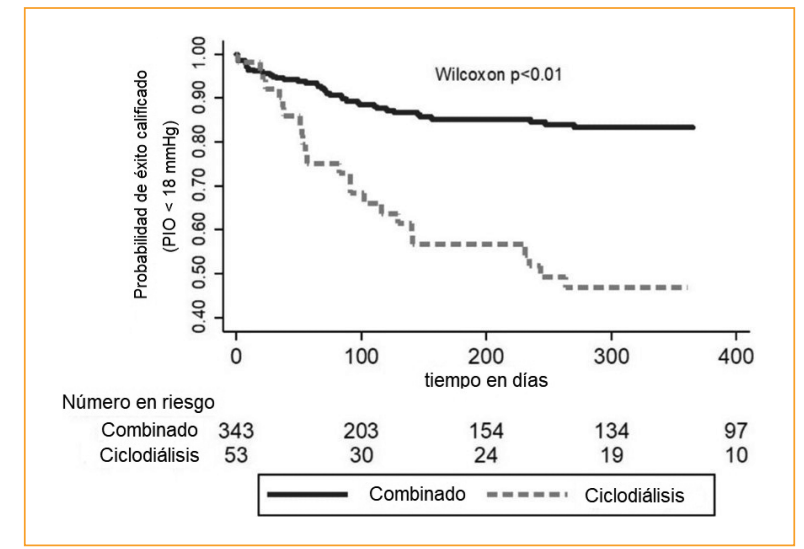

Figura 3. Curva de supervivencia de Kaplan-Meier que muestra la probabilidad acumulada de no fracaso del tratamiento a lo largo del tiempo para ambos grupos y su comparación (presión intraocular [PIO] <18 mmHg): cirugía combinada $(n=343)$ o ciclodiálisis sola $(n=53)$. La diferencia entre ambos grupos está representada por el rango logarítmico de Wilcoxon.

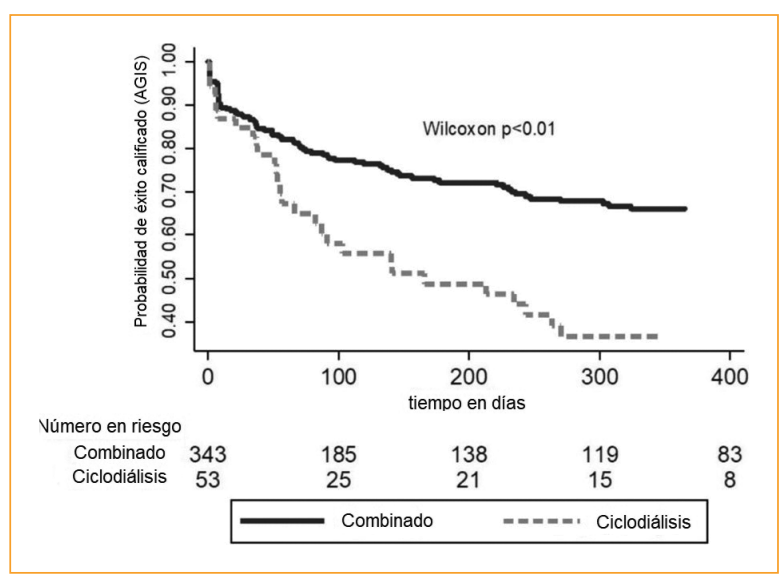

Figura 4. Curva de supervivencia de Kaplan-Meier que muestra la probabilidad acumulada de no fracaso del tratamiento a lo largo del tiempo para ambos grupos y su comparación (presión intraocular $\leq 12.3 \mathrm{mmHg}$ o una reducción del $40 \%$ en casos graves o del $30 \%$ en casos moderados): cirugía combinada $(n=343) 0$ ciclodiálisis sola $(n=53)$. La diferencia entre grupos está representada por el rango logarítmico de Wilcoxon.

Por cada aumento de $1 \mathrm{mmHg}$ en la PIO basal, el riesgo de fracaso aumentaba un 6\% (HR: 1.06; $p<0.01$; IC95\%: 1.03-1.09). En este mismo análisis multivariable no hubo asociación del diagnóstico o la localización de la ciclodiálisis con el desarrollo de fracaso. Estos hallazgos fueron consistentes al aplicar la definición más estricta del AGIS (Tabla 3). 
Tabla 2. Tasas de éxito y éxito calificado durante todo el seguimiento

\begin{tabular}{|c|c|c|c|}
\hline & $\begin{array}{l}\text { mSICS + } \\
\text { ciclodiálisis }\end{array}$ & Ciclodiálisis & p \\
\hline $\begin{array}{l}\text { Éxito }(\mathrm{PIO}<18 \\
\mathrm{mmHg})\end{array}$ & $197(57.4 \%)$ & $15(28.3 \%)$ & $<0.01$ \\
\hline $\begin{array}{l}\text { Éxito calificado } \\
\text { (PIO < } 18 \mathrm{mmHg})\end{array}$ & $289(84.3 \%)$ & $28(52.8 \%)$ & $<0.01$ \\
\hline Éxito AGIS & $166(48.4 \%)$ & $14(26.4 \%)$ & $<0.01$ \\
\hline $\begin{array}{l}\text { Éxito calificado por } \\
\text { AGIS }\end{array}$ & $232(67.6 \%)$ & $24(45.3 \%)$ & $<0.01$ \\
\hline
\end{tabular}

mSICS: cirugía manual de catarata con incisión pequeña; PIO: presión intraocular.
Tabla 4. Complicaciones y reintervenciones

\begin{tabular}{|c|c|c|c|}
\hline Variable & $\begin{array}{l}\text { mSICS + } \\
\text { ciclodiálisis }\end{array}$ & Ciclodiálisis & p \\
\hline \multicolumn{4}{|l|}{ Complicaciones } \\
\hline $\mathrm{RCP}+\mathrm{VTA}$ & $21(6.1 \%)$ & $0(0.0 \%)$ & \multirow[t]{4}{*}{0.03} \\
\hline $\begin{array}{l}\text { Hifema (requirió } \\
\text { tratamiento) }\end{array}$ & $12(3.5 \%)$ & $0(0.0 \%)$ & \\
\hline Hipotonía & $2(0.6 \%)$ & $0(0.0 \%)$ & \\
\hline Otras & $1(0.3 \%)$ & $0(0.0 \%)$ & \\
\hline \multicolumn{4}{|l|}{ Reintervenciones } \\
\hline $\begin{array}{l}\text { Procedimientos para } \\
\text { controlar la PIO }\end{array}$ & $25(7.2 \%)$ & $13(24.5 \%)$ & \multirow[t]{3}{*}{$<0.01$} \\
\hline Lavado de cámara & $15(4.3 \%)$ & $0(0.0 \%)$ & \\
\hline $\begin{array}{l}\text { anterior } \\
\text { Otras }\end{array}$ & $4(1.1 \%)$ & $0(0.0 \%)$ & \\
\hline
\end{tabular}

mSICS: cirugía manual de catarata con incisión pequeña; PIO: presión intraocular RCP: rotura de cápsula posterior; VTA: vitrectomía anterior.

\section{Discusión}

multivariable

\begin{tabular}{|c|c|c|c|c|}
\hline & $\begin{array}{c}\mathrm{PIO}<18 \\
\mathrm{mmHg}\end{array}$ & p & $\begin{array}{l}\text { Definición } \\
\text { AGIS }\end{array}$ & $\mathbf{p}$ \\
\hline $\begin{array}{l}\text { Cirugía combinada } \\
\text { (ref. únicamente } \\
\text { ciclodiálisis) }\end{array}$ & 0.33 & $<0.01$ & 0.50 & $<0.01$ \\
\hline $\begin{array}{l}\text { Diagnóstico (ref. } \\
\text { GPAA) }\end{array}$ & - & - & - & - \\
\hline GCAC & 1.09 & 0.79 & 1.00 & 0.98 \\
\hline PXG & 1.92 & 0.05 & 1.65 & 0.05 \\
\hline Otro* & 1.15 & 0.66 & 1.21 & 0.43 \\
\hline $\begin{array}{l}\text { Localización de la } \\
\text { ciclodiálisis (ref. } \\
\text { SN) }\end{array}$ & - & - & - & - \\
\hline IN & 0.73 & 0.35 & 0.60 & 0.05 \\
\hline ST & 0.65 & 0.30 & 0.64 & 0.15 \\
\hline IT & 1.16 & 0.71 & 0.93 & 0.79 \\
\hline $\begin{array}{l}\text { PIO basal con } \\
\text { tratamiento }\end{array}$ & 1.06 & $<0.01$ & 1.00 & 0.74 \\
\hline
\end{tabular}

GCAC: glaucoma crónico de ángulo cerrado; GPAA: glaucoma primario de ángulo abierto; IN: inferonasal; IT: inferotemporal; PXG: glaucoma por pseudoexfoliación; SN: superonasal; ST: superotemporal.

*Hipertensión ocular, glaucoma secundario a esteroides, glaucoma por mecanismo combinado, glaucoma pigmentario.

\section{Seguridad}

Ocurrieron complicaciones en el $10.5 \%$ del grupo de cirugía combinada, frente al $0 \%$ en el grupo de ciclodiálisis sola $(p=0.03)$. Como se muestra en la tabla 4 , en el grupo de cirugía combinada la mayoría de las complicaciones se relacionaron con la cirugía de catarata (rotura de la cápsula posterior, $6.1 \%$ ). La proporción de reintervención fue mayor en el grupo de ciclodiálisis sola $(p<0.01)$.
Varios estudios de resultados, incluyendo estudios aleatorizados, respaldan que la mSICS es casi tan eficaz, igualmente segura y más rápida de realizar que la facoemulsificación, con un costo sustancialmente menor (US\$ 15.0-15.74 vs. US\$ 42.0-70.0) ${ }^{10,11}$. Por estas razones, la mSICS parece ser la mejor opción en los países en desarrollo.

En nuestra cohorte, la combinación de ciclodiálisis y mSICS se comportó mejor que la ciclodiálisis sola en términos de eficacia. La AVMC mejoró en ambos grupos. El grupo de cirugía combinada logró una mejor AVMC a los 3 meses $(p=0.05)$. En nuestra cohorte de ojos de cirugía combinada, la AVMC se mantuvo sin cambios o mejoró en el $90 \%$ de los casos. Esto es similar a lo observado en un estudio de cirugía combinada realizado por Stark, et al. ${ }^{5}$, en el que la AVMC mejoró en el $93 \%$ de los ojos. Además, el $61 \%$ de los ojos en nuestro grupo de cirugía combinada lograron una AVMC de 0.3 logMar o mejor, que es comparable a lo reportado con cirugía combinada por Mamalis, et al. ${ }^{4}(63 \%)$, Shields y Simmons ${ }^{6}(64 \%)$ y Murchinson $65 \%$, pero menor que en los informes de Ruderman, et al. ${ }^{3}(72 \%)$ y Stark, et al. ${ }^{5}(81 \%)$.

El efecto sobre la reducción de la PIO fue mantenido y estable en ambos grupos, con valores significativamente más bajos a los 12 meses para ambos tipos de cirugía. Sin embargo, la diferencia entre grupos fue significativa. En el último seguimiento, los ojos con cirugía combinada lograron valores más bajos de PIO $(p<0.01)$. En promedio, el porcentaje de reducción de la $\mathrm{PIO}$ en el grupo de cirugía combinada fue del $29 \%$, 
similar a lo reportado por Mamalis, et al. ${ }^{4}(30.8 \%)$ y Ruderman, et al. ${ }^{3}(34 \%)^{3,4}$.

Ambos grupos mostraron una reducción significativa de los requisitos de medicación para el glaucoma $(p<0.01)$. Aunque los ojos del grupo de cirugía combinada utilizaron menos medicamentos preoperatorios, en el seguimiento final la reducción de los requisitos de medicación para el glaucoma fue significativamente mayor en los ojos con cirugía combinada $(p<0.01)$. La reducción media de los requisitos de medicación para el glaucoma en nuestro grupo de cirugía combinada fue de 0.576 ( \pm 1.33 ), lo cual es menor que el 1.44 informado por Mamalis, et al. ${ }^{4}$ en su estudio.

Al comparar las tasas de éxito y de éxito calificado entre ambos grupos, los ojos del grupo de cirugía combinada obtuvieron mejores resultados aplicando ambas definiciones (PIO < $18 \mathrm{mmHg}$ y AGIS) $(p<0.05)$. Una posible razón es que resulta más probable que la hendidura de ciclodiálisis se cierre en los ojos fáquicos que en los pseudofáquicos. De hecho, en nuestra serie, la mayoría de los fracasos en el grupo de ciclodiálisis sola ocurrieron en ojos fáquicos. Aunque la causa es incierta, planteamos la hipótesis de que la extracción de la catarata puede permitir que el iris y el cuerpo ciliar caigan hacia atrás, ejerciendo tensión sobre el espolón escleral y evitando el cierre de la hendidura de ciclodiálisis ${ }^{3}$.

En cirugía combinada (facoemulsificación más trabeculectomía), las tasas de éxito oscilan entre el $67 \%$ y el $78 \%$, y las tasas de éxito calificado oscilan entre el $74 \%$ y el $85 \%(\mathrm{PIO}<21 \mathrm{mmHg})^{4,5,12}$.

Hay pocos informes de cirugía combinada de catarata y ciclodiálisis. En ellos, las tasas de éxito oscilan entre el $60 \%$ y el $83 \%$, y las tasas de éxito calificado oscilan entre el $72 \%$ y el $90 \%$ (PIO $<18 \mathrm{mmHg} 0$ $20 \mathrm{mmHg})^{6,7}$.

En nuestra serie, el éxito calificado para el grupo de cirugía combinada fue del $84.3 \%$ (PIO $<18 \mathrm{mmHg}$ ) y del $67.6 \%$ (AGIS). Cuando comparamos nuestros resultados con otros publicados, son similares incluso al aplicar la definición del AGIS.

El tiempo medio de supervivencia fue significativamente mayor en el grupo de cirugía combinada $(p<0.01)$. El análisis de supervivencia mostró que, cuando se aplicó la definición de PIO $<18 \mathrm{mmHg}$, los ojos en el grupo de cirugía combinada no alcanzaron la mediana del tiempo hasta el fracaso al final del seguimiento. Cuando se aplicó la definición del AGIS, la mediana de tiempo hasta el fallo fue de 747 días (2.05 años). Esto es similar a lo encontrado por Rowan ${ }^{7}$ en un estudio de cirugía combinada, en el que el tiempo medio de supervivencia fue de 34.6 meses (2.8 años); sin embargo, el fracaso en este estudio se definió como PIO > $21 \mathrm{mmHg}$.

En términos de seguridad, el grupo de ciclodiálisis sola mostró mejores resultados $(p=0.03)$. Sin embargo, la mayoría de las complicaciones en el grupo de cirugía combinada se relacionaron con la cirugía de catarata, principalmente en ojos con cataratas hipermaduras, glaucoma por pseudoexfoliación y debilidad zonular conocida. La tasa de rotura de la cápsula posterior en el grupo de cirugía combinada fue del $6.1 \%$, más alta que el $2.8 \%$ reportado por Shields y Simmons ${ }^{6}$ en un estudio de cirugía combinada; sin embargo, estos autores excluyeron de su análisis los ojos con debilidad zonular conocida. Excluyendo estos ojos, la tasa de rotura de la cápsula posterior en nuestra cohorte disminuye al $2.9 \%$. La tasa general de complicaciones en nuestro grupo de cirugía combinada fue del $10.5 \%$, menor que las reportadas en otros estudios de cirugía combinada $(21-36 \%)^{3-7}$.

En este estudio, la proporción de reintervención fue mayor en el grupo de ciclodiálisis sola $(p<0.01)$. La mayoría de las reintervenciones en ambos grupos fueron procedimientos para controlar la PIO. Es posible que la tasa más alta de procedimientos para controlar la PIO en el grupo de ciclodiálisis sola se deba, como mencionamos antes, al cierre de la hendidura de ciclodiálisis en los ojos fáquicos. Al excluir los ojos fáquicos del análisis, la tasa de reintervención es similar en ambos grupos.

En el grupo de cirugía combinada, la tasa general de reintervención fue del $12.6 \%$, similar al $13.8 \%$ informado por Shingleton, et al. ${ }^{13}$ en un estudio de cirugía combinada. En consecuencia, la mayoría de las reintervenciones fueron procedimientos para controlar la PIO.

En nuestro grupo de cirugía combinada, el $2.9 \%$ de los ojos requirieron un lavado de la cámara anterior como tratamiento para el hifema. Otros estudios de cirugía combinada informan una proporción de hifema posoperatorio que oscila entre el $4 \%$ y el $28 \% \frac{3,4,6,7}{\text {. }}$

Este estudio no está exento de limitaciones. El tamaño de la muestra y el seguimiento restringido limitaron el poder estadístico de los resultados. Dado que se recopilaron datos de un solo centro, y que los datos faltantes se manejaron con el método de datos disponibles, podría haber algún sesgo de selección (sin embargo, si el patrón faltante no era completamente aleatorio, los datos faltantes fueron de menos del $3 \%$ ). Se analizaron los datos en busca de cambios en cada ojo intervenido a lo largo del tiempo y, aunque se utilizaron análisis emparejados, podrían existir factores de confusión no 
medidos. Otra limitación de nuestro estudio es que la consideración del daño al nervio óptico y la clasificación del glaucoma se basaron en la evaluación clínica y la PIO preoperatoria (en lugar de en el daño en el campo visual o en los hallazgos de tomografía de coherencia óptica). Por último, es importante mencionar que la patología ocular subyacente distinta del glaucoma, cuando se incluyó en el análisis, influyó en los resultados de la AVMC posoperatoria. Creemos que se deben realizar más estudios prospectivos con un tamaño de muestra más grande e intervenciones aleatorizadas ciegas para permitir el ajuste de otros factores de confusión y extraer conclusiones causales.

En resumen, nuestros resultados proporcionan datos de experiencias de la vida real para ayudar a los cirujanos a decidir si realizar una cirugía combinada en lugar de seguir un enfoque de dos pasos. Nuestro estudio muestra que la combinación de mSICS y ciclodiálisis es un procedimiento eficaz y razonablemente seguro, comparable al método de referencia en términos de tasas de éxito, control de la PIO, reducción de los requisitos de medicamentos para el glaucoma, AVMC y complicaciones. Tomando en cuenta estos hallazgos, la mSICS combinada con ciclodiálisis puede ser particularmente útil en los países en desarrollo.

\section{Financiación}

Esta investigación no recibió ninguna subvención específica de agencias de financiamiento en los sectores público, comercial o sin fines de lucro.

\section{Conflicto de intereses}

No existe conflicto de intereses para ningún autor.

\section{Responsabilidades éticas}

Protección de personas y animales. Los autores declaran que para esta investigación no se han realizado experimentos en seres humanos ni en animales.

Confidencialidad de los datos. Los autores declaran que han seguido los protocolos de su centro de trabajo sobre la publicación de datos de pacientes.

Derecho a la privacidad y consentimiento informado. Los autores declaran que en este artículo no aparecen datos de pacientes.

\section{Anexo}

Anexo 1. Agudeza visual, valores asignados para análisis estadístico.

\begin{tabular}{|l|c|}
\hline Snellen & LogMar \\
\hline $20 / 20$ & 0.00 \\
$20 / 25$ & 0.09 \\
$20 / 30$ & 0.17 \\
$20 / 40$ & 0.30 \\
$20 / 50$ & 0.39 \\
$20 / 60$ & 0.47 \\
$20 / 70$ & 0.54 \\
$20 / 80$ & 0.60 \\
$20 / 100$ & 0.69 \\
$20 / 140$ & 0.84 \\
$20 / 200$ & 1.00 \\
$20 / 400$ & 1.30 \\
CD & 1.80 \\
MM & 1.90 \\
PL & 2.00 \\
NPL & 2.50 \\
\hline
\end{tabular}

CD: counting fingers

MM: hand movement

PL: light perception

NPL: no light perception.

\section{Bibliografía}

1. Bourne RRA, Stevens GA, White RA, Smith JL, Flaxman SR, Price $H$, et al. Causes of vision loss worldwide, 1990-2010: A systematic analysis. Lancet Glob Health. 2013;1:339-49.

2. Mathew RG, Parvizi S, Murdoch IE. Success of trabeculectomy surgery in relation to cataract surgery: 5-year outcomes. $\mathrm{Br} \mathrm{J}$ Ophthalmol. 2019;103:1395-400.

3. Ruderman JM, Fundingsland B, Meyer MA. Combined phacoemulsification and trabeculectomy with mitomycin-C. J Cataract Refract Surg. 1996;22:1085-90.

4. Mamalis N, Lohner S, Rand AN, Crandall AS. Combined phacoemulsification, intraocular lens implantation, and trabeculectomy. J Cataract Refract Surg. 1996;22:467-73.

5. Stark WJ, Goyal RK, Awad O, Vito E, Kouzis AC. The safety and efficacy of combined phacoemulsification and trabeculectomy with releasable sutures. Br J Ophthalmol. 2006;90:146-9.

6. Shields MB, Simmons RJ. Combined cyclodialysis and cataract extraction. Ophthalm Surg. 1976;7:62-73.

7. Rowan PJ. Combined cyclodialysis and cataract surgery. Ophthalm Surg Lasers. 1998;29:962-8.

8. Rotchford AP, King AJ. Moving the goal posts. Definitions of success after glaucoma surgery and their effect on reported outcome. Ophthalmology. 2010;117:18-23.e3.

9. The AGIS Investigators. The Advanced Glaucoma Intervention Study (AGIS): the relationship between control of intraocular pressure and visual field deterioration. Am J Ophthalmol. 2000:130:429-40.

10. Gogate P, Deshpande M, Nirmalan PK. Why do phacoemulsification? Manual small-incision cataract surgery is almost as effective, but less expensive. Ophthalmology. 2007:114:965-8.

11. Ruit S, Tabin G, Chang D, Bajracharya L, Kline DC, Richheimer W, et al. A prospective randomized clinical trial of phacoemulsification vs manual sutureless small-incision cataract surgery in Nepal. Am J Ophthalmology. 2007:143:32-8.

12. Longstaff S, Wormald RPL, Mazover A, Hitchings RA. Glaucoma triple procedures: efficacy of intraocular pressure control and visual outcome. Ophthalmic Surg. 1990;21:786-93.

13. Shingleton BJ, Wooler KB, Bourne $\mathrm{Cl}$, O'Donoghue MW. Combined cataract and trabeculectomy surgery in eyes with pseudoexfoliation glaucoma. J Cataract Refract Surg. 2011;37:1961-70. 